\title{
Depletion of microglia exacerbates injury and impairs function recovery after spinal cord injury in mice
}

\author{
Haitao $\mathrm{Fu}^{1,2}$, Yanpeng Zhao ${ }^{1}$, Die $\mathrm{Hu}^{3}$, Song Wang ${ }^{4}$, Tengbo $\mathrm{Yu}^{2}$ and Licheng Zhang ${ }^{1}$
}

\begin{abstract}
The role of microglia in spinal cord injury (SCl) remains ambiguous, partially due to the paucity of efficient methods to discriminate these resident microglia with blood-derived monocytes/macrophages. Here, we used pharmacological treatments to specifically eliminate microglia and subsequently to investigate the response of microglia after $\mathrm{SCl}$ in mice. We showed that treatment with colony stimulating factor 1 receptor (CSF1R) inhibitor PLX3397 eliminated 90\% microglia and did not affect other cell types in mouse spinal cord. PLX3397 treatment also induced a strong decrease in microglial proliferation induced by SCl. Depletion of microglia after $\mathrm{SCl}$ disrupted glial scar formation, enhanced immune cell infiltrates, reduced neuronal survival, delayed astrocyte repopulation, exacerbated axonal dieback, and impaired locomotor recovery. Therefore, our findings suggest microglia may play a protective role after SCl in mice.
\end{abstract}

\section{Introduction}

Microglia are derived from primitive myeloid progenitors in the yolk sac during embryonic development ${ }^{1}$. As the main immune cells in central nervous system (CNS), microglia help to sculpt the mature CNS during development by removing apoptotic cells and inappropriate neural connections ${ }^{2}$. In the adult CNS, microglia constantly surveil the microenvironment for alterations resulting from injury or disease $\mathrm{e}^{2,3}$. After sensing perturbations, microglia become activated, reorient their processes towards the lesion, and phagocytose cellular debris ${ }^{4}$.

However, the role of microglia in CNS injury remains controversial. Activated microglia release proinflammatory factors that cause neuronal death and contribute to the secondary tissue damage ${ }^{2,5}$. Inhibition of microglia proliferation reduces inflammatory response, alleviates neuronal death, and improves motor recovery

Correspondence: Tengbo Yu (ytb8912@163.com) or

Licheng Zhang (zhanglcheng218@126.com)

'Department of Orthopedics, Chinese PLA General Hospital, Beijing, China

2Department of Orthopaedics, The Affiliated Hospital of Qingdao University,

Qingdao, China

Full list of author information is available at the end of the article.

Edited by K. Blomberg after spinal cord injury $(\mathrm{SCI})^{6,7}$. Conversely, several studies demonstrate beneficial roles of microglia in CNS injury. In a mouse model of stroke, microglia are proved to play a role in protecting neurons by regulating intracellular calcium levels ${ }^{8}$. In a mouse model of contusive $\mathrm{SCI}$, microglia are identified as a key cellular component of the scar that develops after SCI to protect neural tissue $^{9}$. In addition, one study show that microglia are irrelevant for neuronal degeneration and axon regeneration after acute crush injury of optic nerve ${ }^{10}$. Therefore, microglia may exert diverging roles depending on the context. Whether microglia are beneficial or detrimental for recovery after SCI remains unclear.

In addition to their potentially conflicting roles, the paucity of efficient methods to distinguish these resident microglia with blood-derived monocytes/ macrophages hamper the exploration of the specific roles of microglia after a CNS injury ${ }^{11}$. The newly developed pharmacologic strategies based on CSF1R inhibition specifically eliminate $\sim 99 \%$ microglia in adult brain, whereas peripheral macrophages and other immune cells remained unaffected $^{10,12}$. This method allows the study of the specific roles of microglia in CNS injury.

\section{(c) The Author(s) 2020}

(c) (i) Open Access This article is licensed under a Creative Commons Attribution 4.0 International License, which permits use, sharing, adaptation, distribution and reproduction cc) in any medium or format, as long as you give appropriate credit to the original author(s) and the source, provide a link to the Creative Commons license, and indicate if changes were made. The images or other third party material in this article are included in the article's Creative Commons license, unless indicated otherwise in a credit line to the material. If material is not included in the article's Creative Commons license and your intended use is not permitted by statutory regulation or exceeds the permitted use, you will need to obtain permission directly from the copyright holder. To view a copy of this license, visit http://creativecommons.org/licenses/by/4.0/. 
Here, we used PLX3397, a CSF1R inhibitor that specifically eliminate microglia to investigate the specific roles of microglia in spinal cord. We showed that PLX3397 treatment eliminated almost all microglia and the absence of microglia did not affect other cell types in spinal cord. Depletion of microglia in the context of SCI was associated with disorganized astroglial scar, reduced neuronal number, delayed astrocyte repopulation, aggravated axonal dieback, and reduced functional recovery. Therefore, microglia may have a beneficial effect on function recovery after SCI.

\section{Materials and methods}

\section{Animal experiments}

Female C57BL/6 mice (6-week-old) were used for all experiments. Mice were housed under controlled conditions with $12 \mathrm{~h}$ light/dark cycle and had free access to food and water at all time. All animal procedures were approved by the Institutional Animal Care and Use Committee of the Chinese PLA General Hospital and conducted in accordance with relevant guides of the Chinese Ministry of Public Health on the care and use of laboratory animals and in compliance with the ARRIVE (Animal Research: Reporting In Vivo Experiments) guidelines.

\section{Microglia depletion}

To deplete microglia in vivo, mice were given the CSF1R inhibitor PLX3397 (pexidartinib; Selleckchem, Houston, TX, USA) formulated into AIN-76A standard diet at $290 \mathrm{mg} / \mathrm{kg}$ for 7 days $^{5}$. AIN-76A standard diet was used as respective controls.

\section{Spinal cord injury}

A full crush injury was performed similar to that previously described by Liu et al. ${ }^{13}$. Mice were anesthetized by intraperitoneal injections of sodium pentobarbital at $80 \mathrm{mg} / \mathrm{kg}$. A midline incision was made over the thoracic vertebrae. Then, a laminectomy was conducted at the level of T10 segment until the spinal cord was exposed completely from side to side. The exposed spinal cord was then crushed for $2 \mathrm{~s}$ with modified forceps. The forceps were filed to a width of $0.1 \mathrm{~mm}$ for the last $4-5 \mathrm{~mm}$ of the tips. The spinal dura was intact after crushing. The muscle and skin were sutured. Post-operatively, mice were placed on a warming blanket until fully awake. Bladders were emptied manually twice daily to prevent urinary tract infections until mice were able to urinate independently.

\section{Automated capillary western blot (WES)}

Mice were transcardially perfused with cold phosphatebuffered saline (PBS) and then the spinal cords were isolated from spinal columns for western blot analysis. Western blots were performed using WES, an automated capillary-based size sorting system (ProteinSimple, San Jose, CA, USA) ${ }^{14}$. All procedures were performed according to the manufacturer's protocol. Briefly, $8 \mu \mathrm{L}$ of diluted protein lysate was mixed with $2 \mu \mathrm{L}$ of $5 \times$ fluorescent master mix and heated at $95^{\circ} \mathrm{C}$ for $5 \mathrm{~min}$. The samples, blocking reagent, wash buffer, primary antibodies, secondary antibodies, and chemiluminescent substrate were dispensed into designated wells in a microplate provided by the manufacturer. The plate was loaded into the instrument and protein was drawn into individual capillaries on a 25 capillary cassette provided by the manufacturer. Protein separation and immunodetection was performed automatically on the individual capillaries using default settings. The data was analyzed with Compass software (ProteinSimple). Primary antibodies used were anti-Iba1 antibody (abcam, ab153696), anti-ßIII Tubulin antibody (abcam, ab78078), anti-GFAP antibody (abcam, ab4674), and anti-Olig2 antibody (abcam, ab109186).

\section{BDA tracing}

This procedure is similar to what was described previously with modifications ${ }^{13,15}$. A midline incision was made over the skull to reveal bregma after mice were anesthetized. Then, a window in the skull was made with a microdrill to expose the sensorimotor cortex. To label corticospinal tract (CST) axons by anterograde tracing, four injections of $500 \mathrm{nl}$ BDA (10\%, Invitrogen, D1956) were injected into sensorimotor cortex at four sites through a glass pipette attached to a nanoliter injector at a rate of $1 \mathrm{nl} / \mathrm{s}$. The four coordinates were as follows: $1.5 \mathrm{~mm}$ lateral, $0.6 \mathrm{~mm}$ deep, and $0.5 \mathrm{~mm}$ anterior; $0.0 \mathrm{~mm}, 0.5 \mathrm{~mm}$, and $1.0 \mathrm{~mm}$ caudal to bregma. In order to prevent the backflow of the injection, the needle was kept in situ for $1 \mathrm{~min}$ before moving to the next site. The skin was sutured and mice were then placed on a warming blanket. These BDA-injected mice were kept for an additional 2 weeks before termination.

\section{Tissue processing}

For the purpose of histology and immunofluorescence experiments, mice were anesthetized and transcardially perfused with cold PBS followed by 4\% paraformaldehyde (PFA). Spinal columns were isolated and post-fixed in the $4 \%$ PFA overnight at $4{ }^{\circ} \mathrm{C}$. A spinal cord segment of $10 \mathrm{~mm}$ containing the lesion site were dissected out and then immersed for 3 days at $4{ }^{\circ} \mathrm{C}$ in a PBS solution containing 30\% sucrose for cryoprotection. Tissues were then embedded in OCT compound, snap-frozen in dry ice and stored at $-20^{\circ} \mathrm{C}$ until processed.

\section{HE and immunofluorescence stain}

For HE stain, coronal sections of the spinal cord centered over the lesion site were cut on a cryostat at 
25-30 $\mu \mathrm{m}$ and then stained using a commercial staining kit (Solarbio, G1120). For Immunofluorescence stain, coronal, sagittal, or transverse sections of the spinal cord were cut at $25-30 \mu \mathrm{m}$ thickness. All steps of immunostaining were performed following standard protocols. Briefly, sections were rinsed with PBS for $5 \mathrm{~min}$ at room temperature, encircled with a hydrophobic barrier, and blocked for $2 \mathrm{~h}$ with $10 \%$ normal goat serum, $0.5 \%$ Triton $\mathrm{X}-100$ in PBS. Primary antibodies used in this study are of the following sources and were used at the indicated dilutions: rabbit anti-Iba1 (1:1000, Wako, 019-19741), Chicken anti-GFAP (1:1000, Abcam, ab4674), rabbit antiOlig2 (1:200, Abcam, ab109186), mouse anti-BIII Tubulin (1:300, Abcam, ab78078), rabbit anti-CD45 (1:500, Abcam, ab10558), rabbit anti-NeuN (1:300, Abcam, ab177487). Secondary antibodies conjugated to Alexa488, Alexa-594, or Alexa-647 were from abcam and diluted 1/500. Sections were incubated with primary antibody in a humidified chamber overnight at $4{ }^{\circ} \mathrm{C}$, rinsed three times in PBS, incubated with secondary antibody for $2 \mathrm{~h}$ at room temperature, rinsed three times in PBS, incubated with DAPI (1/200 in PBS, Solarbio, C0060), rinsed twice in PBS. Finally, coverslips were mounted. For BDA labeling, Alexa Fluor ${ }^{\circledR} 594$ streptavidin conjugate (1/200 in PBS, Invitrogen, S32356) was added to the secondary antibody solution and incubated with secondary antibodies.

\section{Quantitative analyses}

Quantification of stained tissue sections was performed by Image Pro Plus (IPP) software. For the quantification of microglia (Iba1+), astrocytes (GFAP + ), oligodendrocytes (Olig2+), the total number of immunolabeled cells per cross section was counted at $\times 10$ magnification immunofluorescence images obtained using a microscope equipped with a DP71 camera. For the quantification of neurons ( $\beta$ III Tubulin ${ }^{+}$), the number of immunolabeled cells in $400 \times 300 \mu \mathrm{m}$ sectors from spinal cord ventral horn was counted at $\times 20$ magnification immunofluorescence images. For the quantification of cell body size of astrocytes or microglia, the longest diameter of cell body of one astrocyte or microglia per cross section was measured at $\times 20$ magnification images. For the quantification of process lengths of astrocytes, the longest process of one astrocyte per cross section was measured at $\times 20$ magnification images. The length of longest process was the linear distance from the initial part to farthest end of the process. For the quantification of lesion area, lesion site was manually outlined according to anti-GFAP immunofluorescence and quantified by IPP software. For the quantification of fluorescence intensity of CD45+ cells surrounding the lesion, Mean gray values of CD45 staining is determined in three randomly selected $150 \times 150 \mu \mathrm{m}$ sectors around the lesion and the average represent the mean fluorescence intensity per sample. For the quantification of axonal dieback distance, the vertical distance from the farthest end of BDA+ process to vertical line of spinal cord long axis, which pass through the lesion epicenter was measured at $\times 10$ magnification images.

\section{In vivo T2-weighted magnetic resonance imaging (T2W- MRI)}

T2W-MRI acquisitions were done at 3 weeks after injury using a small animal-designed 7.0 T MRI scanner (Bruker, PharmaScan; Bruker BioSpin, Germany) with a $38-\mathrm{mm}$ quadrature volume coil was used. Mice were anesthetized (1.5\% isoflurane) using a MR-compatible anesthetic equipment and T2 sequences were conducted using the Bruker ParaVision 5.0 system. After that, mice were placed back warm cages.

\section{Behavioral analysis}

Recovery of locomotor function after SCI was quantified in an open field using the Basso Mouse Scale (BMS), according to the method developed by Basso and colleagues $^{16}$. The BMS is a 10-point locomotor rating scale, in which 9 point means normal locomotion while 0 equals to complete paralysis. Two investigators, who are blinded to the experiment, independently gave the score of the mice.

\section{Statistics}

Statistical significances were evaluated using Student's $t$-test, one-way or two-way ANOVA. Two-tailed unpaired Student $t$-test was used to determine the significance of differences between two groups. One-way ANOVA followed by Tukey post hoc test was used for comparisons of three or more groups. Two-way ANOVA followed by Bonferroni post-tests was used for multiple comparisons. All statistical analyses were performed using the GraphPad Prism software version 5.0 (GraphPad Software Inc.). Values were presented as mean \pm standard deviation(SD). A $p$-value $<0.05$ was considered as statistically significant.

\section{Results}

Efficient depletion of microglia in the adult spinal cord

Diet-mediated application of the CSF1R inhibitor PLX3397 was shown previously to deplete microglia efficiently in mouse adult brains and optic nerves ${ }^{5,12}$. As spinal cords were not analyzed in these studies, we first addressed this aspect by processing these spinal cords from mice on normal or PLX3397 diet for the expression of Iba1, a marker for microglia. Spinal cords were homogenized, and western blots were performed. As expected, mice with 7-day PLX3397 treatment were found to have decreased levels of Iba1. The Iba1 protein levels decreased to $95 \%$ below the levels of the control diet 


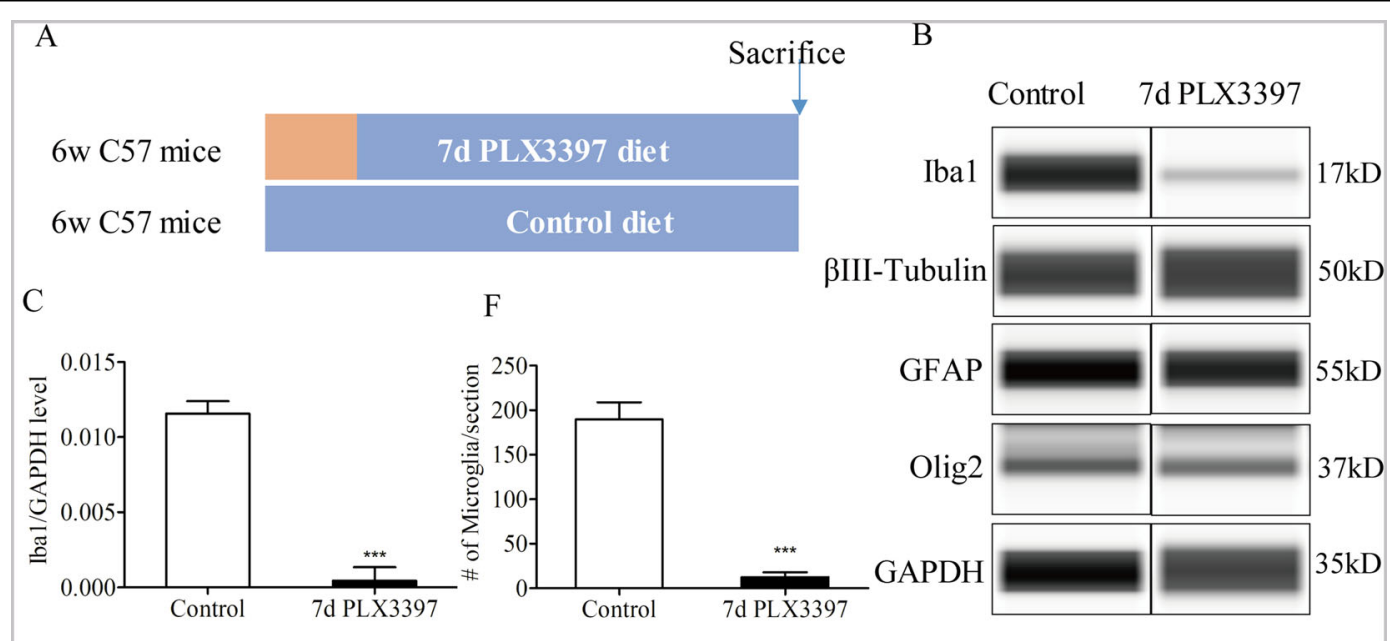

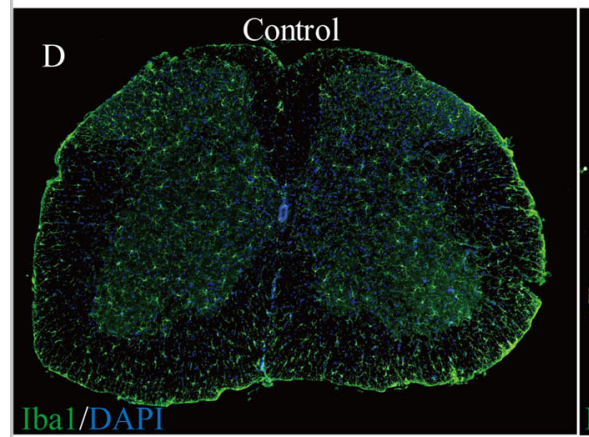

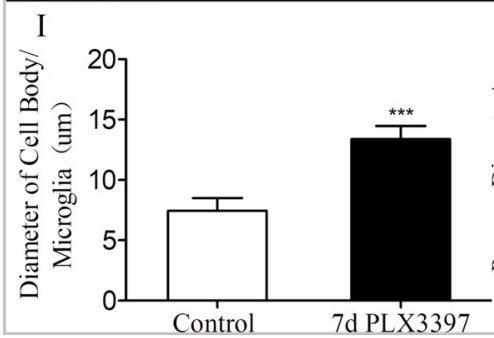

$\mathrm{J}$

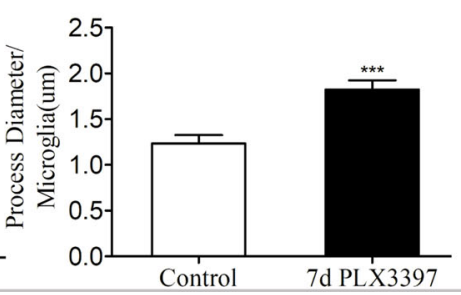

7d PLX3397

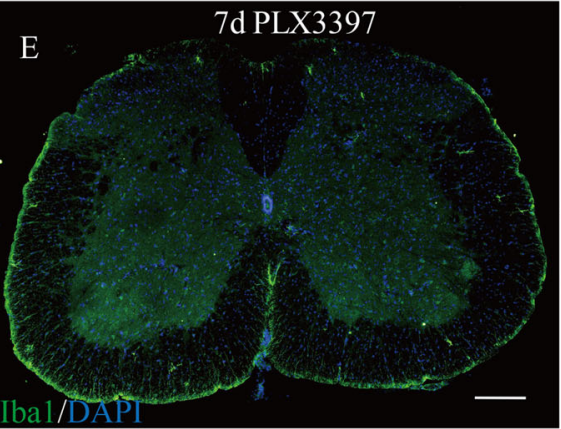

$\mathrm{K}$

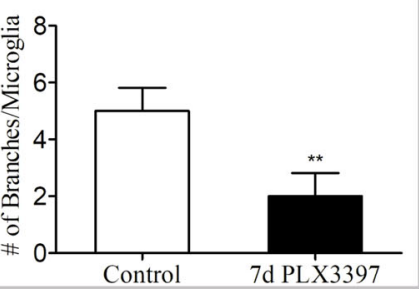

Fig. 1 Efficient depletion of microglia in the adult spinal cord via pharmacologic inhibition of CSF1R. a Schematic of the experimental design: 6-week-old C57BL/6 mice were fed either PLX3397 or control chow for 7 days. On day 7, mice were euthanized for western blots or Immunostaining. b Western blot analysis of spinal cord homogenates for steady state levels of the microglia marker Iba1, neuronal markers $\beta$ III Tubulin, the oligodendrocyte marker Olig2, and the astrocyte markers GFAP. c Quantification of Iba1 in (B) showing CSF1R inhibition decreased Iba1 protein levels ( $n=4$ per group). d, e Representative immunofluorescence images of mouse spinal cord sections showing Iba ${ }^{+}$ramified microglia (green). Almost no $\mid \mathrm{ba} 1^{+}$cells were detected in mice that were fed a PLX3397 diet for $7 \mathrm{~d}$. $\mathbf{f}$ Quantification of the number of $\mathrm{IBA} 1^{+}$cell in the spinal cord from control and PLX3397-treated mice ( $n=4$ per group) as shown in (D) and (E). $\mathbf{g}, \mathbf{h}$ Iba1 immunostaining shows changes in microglia morphology with representative microglia shown from control and 7-day PLX3397-treated mice. I-K Microglial morphology were assessed by the diameter of cell body per microglia (i), the process diameter per microglia (j) and the number of branches per microglia (k) $(n=4$ per group). Data are expressed as mean \pm SD. Scale bars: $\mathbf{d}$, e, in e $200 \mu \mathrm{m} ; \mathbf{g}, \mathbf{h}$, in $\mathbf{h} 10 \mu \mathrm{m}$.

group (Fig. 1b, c). Immunostaining for Iba1 in the spinal cord of these animals confirmed the results and further revealed a clear decrease in microglia numbers with PLX3397 treatments (Fig. 1d-f). Only single remaining cells were very occasionally found with a larger cell body, an increased thickness of processes, and a reduction in the number of branches (Fig. 1g-k), typically associated with a more phagocytotic phenotype ${ }^{17}$. These data indicate that PLX3397 can be used to selectively and nearly completely eliminate spinal cord microglia under steady state in vivo conditions in mice.

\section{Microglial depletion does not affect other cell types in spinal cord}

To explore whether microglial depletion could affect other cell types in spinal cord, we test protein expression levels of neuronal, oligodendrocytic, and astroctytic markers via western blot (Fig. 1b) No changes in markers 


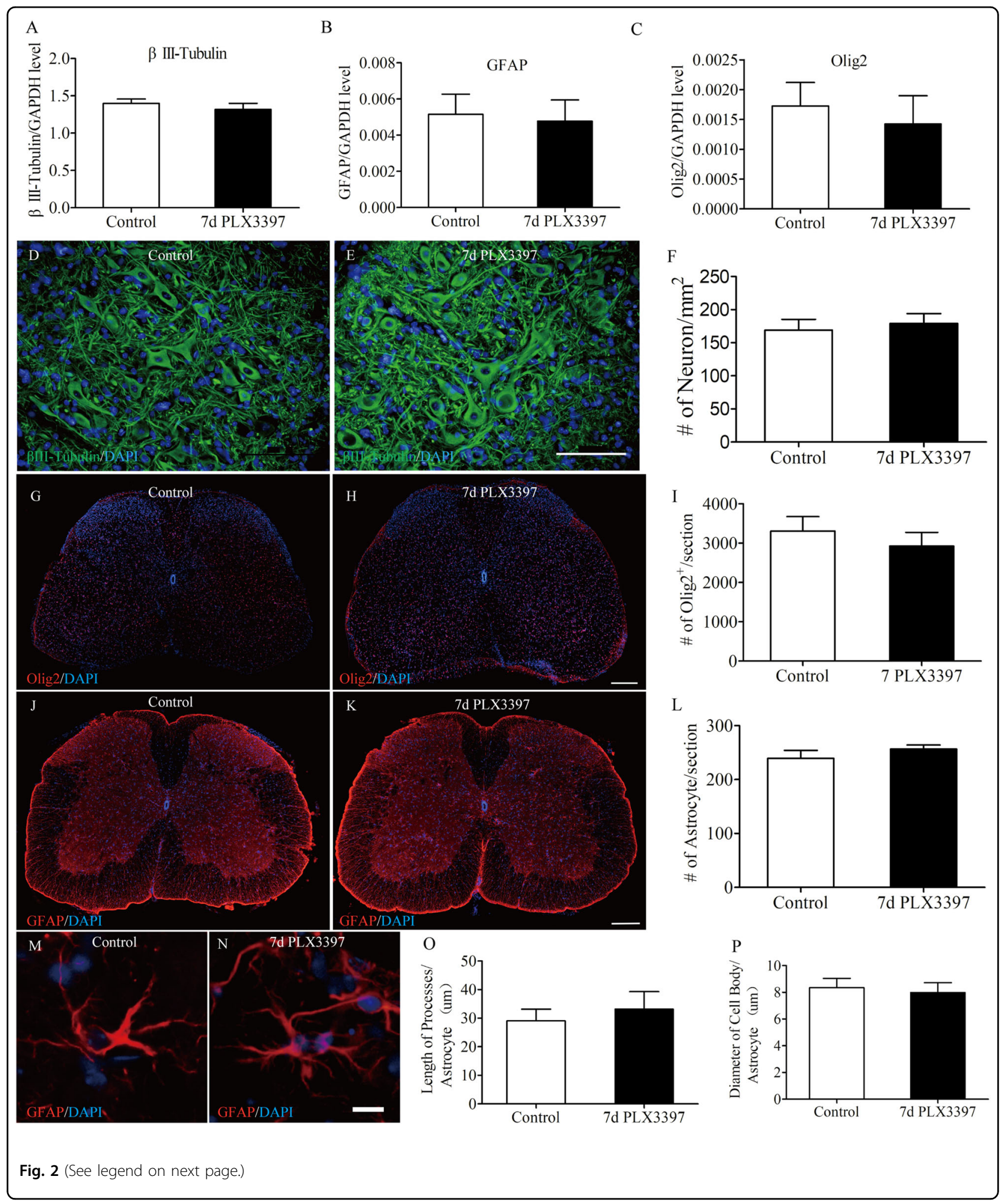

BIII Tubulin, oligodendrocyte transcription factor (Olig2), or glial fibrillary acidic protein (GFAP) were observed (Fig. 2a-c). Immunofluorescent stains for $\beta$ III Tubulin,
Olig2 or GFAP in the spinal cord of these mice confirmed the results. BIII Tubulin+, Olig2+ or GFAP + cell counts showed no differences with microglial depletion (Fig. 2d-1). 
(see figure on previous page)

Fig. 2 Microglia elimination does not affect other cell types in spinal cord. Six-week-old wild-type C57BL/6 mice were treated with PLX3397 or vehicle or 7 days to eliminate microglia. Western blots were performed on spinal cord homogenates for the neuronal markers $\beta$ III Tubulin, the oligodendrocyte marker Olig2, and the astrocyte markers GFAP (Fig. 1b). a-c Quantification of Blll Tubulin, Olig2, and GFAP in Fig. 1b shows no significant changes in the expression of the three markers between two groups ( $n=4$ per group). $\mathbf{d}$, e Representative images taken from mouse spinal cord ventral horn immunostained for $\beta$ III-Tubulin (green) and DAPI (blue). $\mathbf{f}$ Quantification of the number of $\beta$ III-Tubulin ${ }^{+}$cells in the spinal cord from control and PLX3397-treated mice ( $n=4$ per group) as shown in $\mathbf{d}$ and $\mathbf{e} . \mathbf{g}, \mathbf{h}$ Representative immunofluorescence images of mouse spinal cord sections showing Olig2 ${ }^{+}$cells. i Quantification of the number of Olig2 ${ }^{+}$cells in the spinal cord from control and PLX3397-treated mice $(n$ $=4$ per group) as shown in $\mathbf{g}$ and $\mathbf{h}$. j, $\mathbf{k}$ Representative immunofluorescence images of mouse spinal cord sections (the same sections with Fig. 1d, e, respectively) showing GFAP ${ }^{+}$cells. I Quantification of the number of GFAP ${ }^{+}$cells in the spinal cord from control and PLX3397-treated mice $(n=4$ per group) as shown in $\mathbf{j}$ and $\mathbf{k}$. $\mathbf{m}, \mathbf{n}$ GFAP immunostaining shows the morphology of astrocytes from control and 7-day PLX3397-treated mice. $\mathbf{0}, \mathbf{p}$ Astrocyte morphology were assessed by the length of processes per astrocyte and the diameter of cell body per astrocyte ( $n=4$ per group). Data are expressed as mean \pm SD. Scale bars: $\mathbf{d}$, e in e $100 \mu \mathrm{m} ; \mathbf{g}, \mathbf{h}$ in $\mathbf{h} 200 \mu \mathrm{m} ; \mathbf{j}, \mathbf{k}$ in $\mathbf{k} 200 \mu \mathrm{m} ; \mathbf{m}, \mathbf{n}$ in $\mathbf{n} 10 \mu \mathrm{m}$.

Given the important crosstalk between astrocytes and microglia, we further performed morphological analyses of astrocytes with 7 or 14 days of PLX3397 treatment. The results revealed that no changes in astrocyte cell body sizes or process lengths after microglial depletion (Fig. $2 \mathrm{~m}-\mathrm{p}$ and Supplementary Fig. 1). Therefore, elimination of microglia results in no changes in cell numbers or morphology.

\section{CSF1R blockade reduce microglial accumulation induced by $\mathrm{SCl}$}

To explore the effect of microglia depletion in SCI context, wild-type C57BL/6 mice were fed with PLX3397 or control diet for 7 days prior to sham-operation (only performed laminectomy) or SCI procedures (Fig. 3a). Thereafter, these mice continued to receive PLX3397 or control diet until 7 days post injury (dpi). The spinal cords of these mice were isolated form spinal columns for immunofluorescent stains or western blot. Western blot analysis of spinal cord homogenates showed a robust increase in levels of Iba1 expression at 7 days after full crush injury. Treatment with CSF1R inhibition prevented this SCI-induced Iba1 increase (Fig. 3b, c). Immunofluorescent stains for Iba1 in the spinal cord of mice fed with control diet showed that microglia accumulated around the lesion site at $7 \mathrm{dpi}$ and exhibited a round morphology, which points to a potential increase in their phagocytic activity. In the spinal cord of mice with PLX3397 treatment, microglia around the lesion epicenter dramatically reduce at 7 days following SCI (Fig. $3 d-f$ ). Thus, our data indicate that microglia are rapidly recruited around the site of SCI and CSF1R blockade reduce this accumulation induced by SCI.

\section{Microglial depletion reduces locomotor recovery after SCI}

We next investigated the role of microglia in functional recovery after SCI. Chow containing either PLX3397 or no drug was given to C57BL/6 mice starting 7 days prior to SCI and then maintained for an additional 4 weeks (Fig. 4a). The effect of PLX3397-mediated CSF1R inhibition and reduced microglial accumulation on motor behavior following SCI was evaluated using BMS scores. We showed that mice depleted of microglia exhibited impaired locomotor recovery compared to mice treated with control diet at 7, 14, 21, and $28 \mathrm{dpi}$ (Fig. 4b). Thus, the results suggest that microglia play an essential role in motor function recovery after SCI.

\section{Microglial depletion disorganizes astrocytic scar formation}

In cases of injury to the spinal cord, a key pathological event is the formation of a glial scar induced by reactive astrogliosis ${ }^{18}$. Whether scar formation contributes to functional recovery following CNS injury remains a matter of debate. Recently, Anderson and his colleagues ${ }^{19}$ showed that astrocyte scar formation aids CNS axon regeneration. Thus, we next explored whether microglia play an important role in the formation of the astrocytic scar that develops following SCI. Strikingly, both HE and Immunofluorescence stain showed astrocytes around the lesion core formed a less compact scar when microglia were depleted using PLX3397 compared to the control treatment at $30 \mathrm{dpi}$ (Fig. 5a-f). Notably, we observed that GFAP + astrocytes were oriented randomly and not aligned in any particular direction in PLX3397-treated SCI mice at $21 \mathrm{dpi}$ (Fig. 5g, h). This disorganized astroglial scar was accompanied by an increased infiltration of $\mathrm{CD} 45^{+}$cells into the spinal cord parenchyma (Fig. 5i-k), which may lead to an increase of intramedullary highsignals in SCI mice with PLX3397 treatment (Fig. 5l, m).

\section{Microglial depletion delays the repopulation of the lesion site by astrocytes after $\mathrm{SCl}$}

It was proposed that reactive astrocytes exert protective functions after $\mathrm{SCI}^{20}$. We next investigated whether microglial depletion affects tissue damage. Immunofluorescence staining for GFAP revealed that the lesion area significantly increased in PLX3397-treated mice compared to that in control treated mice at 7 days post SCI, because a larger, seemingly cell-free area with strongly reduced GFAP staining was observed in these 
A

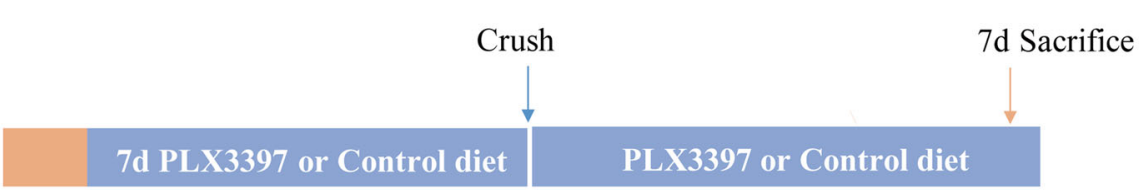

6w C57 mice

B

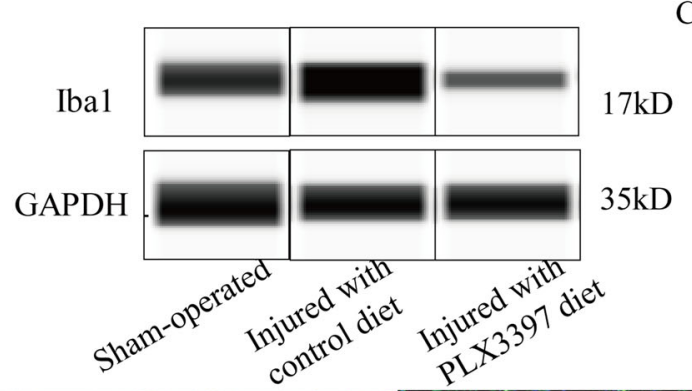

$\mathrm{C}$

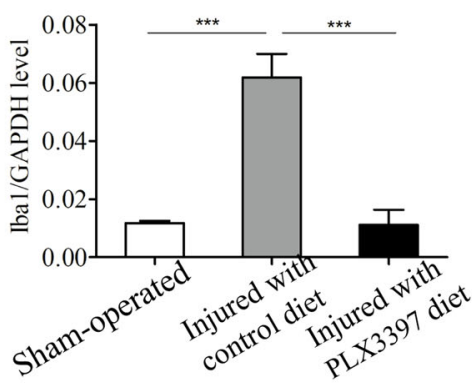

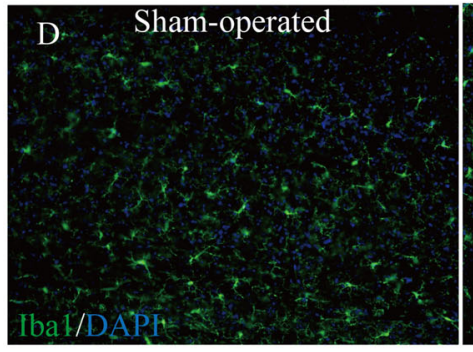
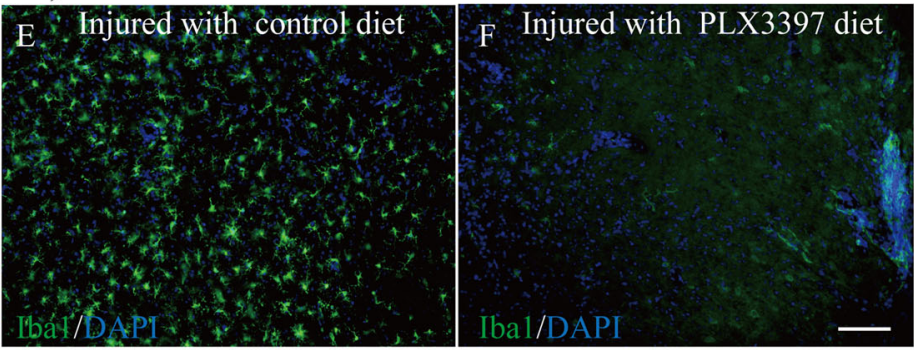

Fig. 3 CSF1R inhibition reduces microglial accumulation induced by SCI. a Schematics of experimental design showing the timeline of microglia depletion, spinal cord crush injury, and sacrifice. $\mathbf{b}$ Western blot analysis of spinal cord homogenates from sham-operated, injured with control or PLX3397-treated mice ( $n=4$ per group) for steady state levels of the microglia marker Iba1. c Quantification of $\mathbf{b}$ showing a robust increase in levels of Iba1 expression at 7 days following SCI. CSF1R inhibition reverses the increase induced by SCl. $\mathbf{d}-\mathbf{f}$ Representative immunofluorescence images of mouse spinal cord sections showing $\mathrm{Iba} 1^{+}$microglia. The changes in number of $\mathrm{Iba} 1^{+}$microglia consist with the changes of Iba1 expression levels. Data are expressed as mean \pm SD. Scale bars: e, $\mathbf{f}$ in $\mathbf{f} 100 \mu \mathrm{m}$.
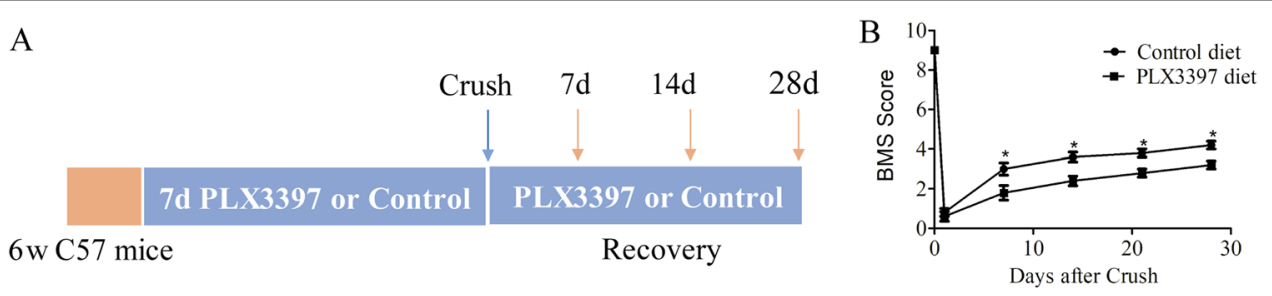

Fig. 4 Microglial depletion reduces locomotor recovery after SCI. a Schematics of experimental design showing the timeline of microglia depletion, spinal cord crush injury, behavioral testing using the BMS score. $\mathbf{b}$ Locomotor function was assessed using the BMS score over a 28-day period after $\mathrm{SCl}$ ( $n=4$ per group). Data are expressed as mean \pm SD. ${ }^{*} p<0.05$.

PLX3397-treated spinal cord (Fig. 6a, d). Quantification revealed that microglial depletion was correlated with an increase of the lesion core area at $7 \mathrm{dpi}$. However, no significant differences in lesion area between groups were detected at 14 and $30 \mathrm{dpi}$, although lesion sizes tended to be slightly larger in PLX3397-treated mice (Fig. 6g). Thus, microglia seem to have a rather transient role in this process and their absence just delayed astrocyte repopulation of the lesion site.

\section{Microglial depletion reduces neuronal survival and exacerbates axonal dieback}

Having established that the lesion area was increased at $7 \mathrm{dpi}$ and functional recovery worse at $7,14,21$, and $28 \mathrm{dpi}$ in microglia-depleted mice, we next investigated whether microglial depletion would influence neuronal survival. Immunofluorescence staining for NeuN revealed that there were fewer neurons around lesion epicenter in spinal cord sections of PLX3397 diet group compared 


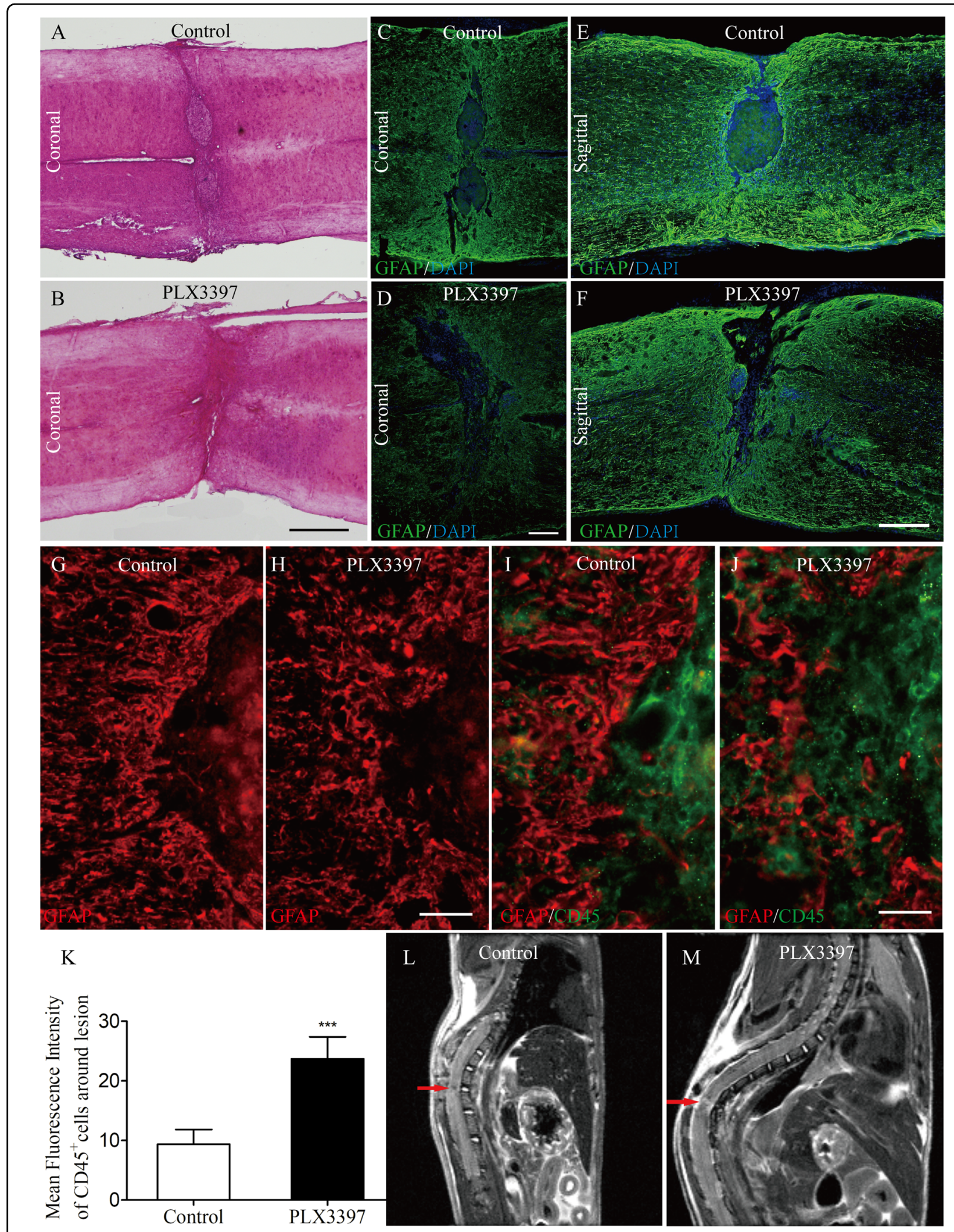

Fig. 5 (See legend on next page.) 
(see figure on previous page)

Fig. 5 The elimination of microglia results in a disorganized astrocytic scar at the lesion border. $\mathbf{a}-\mathbf{f}$ Representative HE $(\mathbf{a}, \mathbf{b})$ or immunofluorescence $(\mathbf{c}-\mathbf{f})$ images of coronal $(\mathbf{a}-\mathbf{d})$ or sagittal $(\mathbf{e}, \mathbf{f})$ sections of mouse spinal cord taken at $30 \mathrm{dpi}$. In control mice $(\mathbf{a}, \mathbf{c}, \mathbf{e})$, astrocytes adjacent to the lesion form a compact scar. This astrocytic scar was compromised in mice depleted of microglia using PLX3397 (b, $\mathbf{d}, \mathbf{f})$. $\mathbf{g}-\mathbf{j}$ Representative immunofluorescence images of spinal cord sections taken at the lesion epicenter at $21 \mathrm{dpi}$. In mice fed with the control diet (g, i), astrocytes exhibit elongated processes oriented parallel to the lesion border. This orientation was disorganized in PLX3397-treated mice (h, $\mathbf{j})$ and associated with clusters of $\mathrm{CD}_{4} 5^{+}$immune cells spreading outside of the lesion epicenter. $\mathbf{k}$ Quantification of the infiltration degree of CD45+ cells spreading outside of the lesion epicenter in the spinal cord from control and PLX3397-treated mice ( $n=4$ per group) as shown in $\mathbf{i}$ and $\mathbf{j}$. I, $\mathbf{m}$ In vivo T2W-MRI assessment of the lesion in control and PLX3397-treated mice after SCl. In vivo sagittal sections of mouse spinal cord taken at $21 \mathrm{dpi}$. Red arrows represent abnormal signals in the spinal cord. Scale bars: $\mathbf{a}, \mathbf{b}$, in $\mathbf{b} 1 \mathrm{~mm} ; \mathbf{c}, \mathbf{d}$, in $\mathbf{d} 200 \mu \mathrm{m} ; \mathbf{e}, \mathbf{f}$, in $\mathbf{f} 200 \mu \mathrm{m} ; \mathbf{g}, \mathbf{h}$, in $\mathbf{h} 50 \mu \mathrm{m} ; \mathbf{i}, \mathbf{j}$, in $\mathbf{j} 25 \mu \mathrm{m}$.
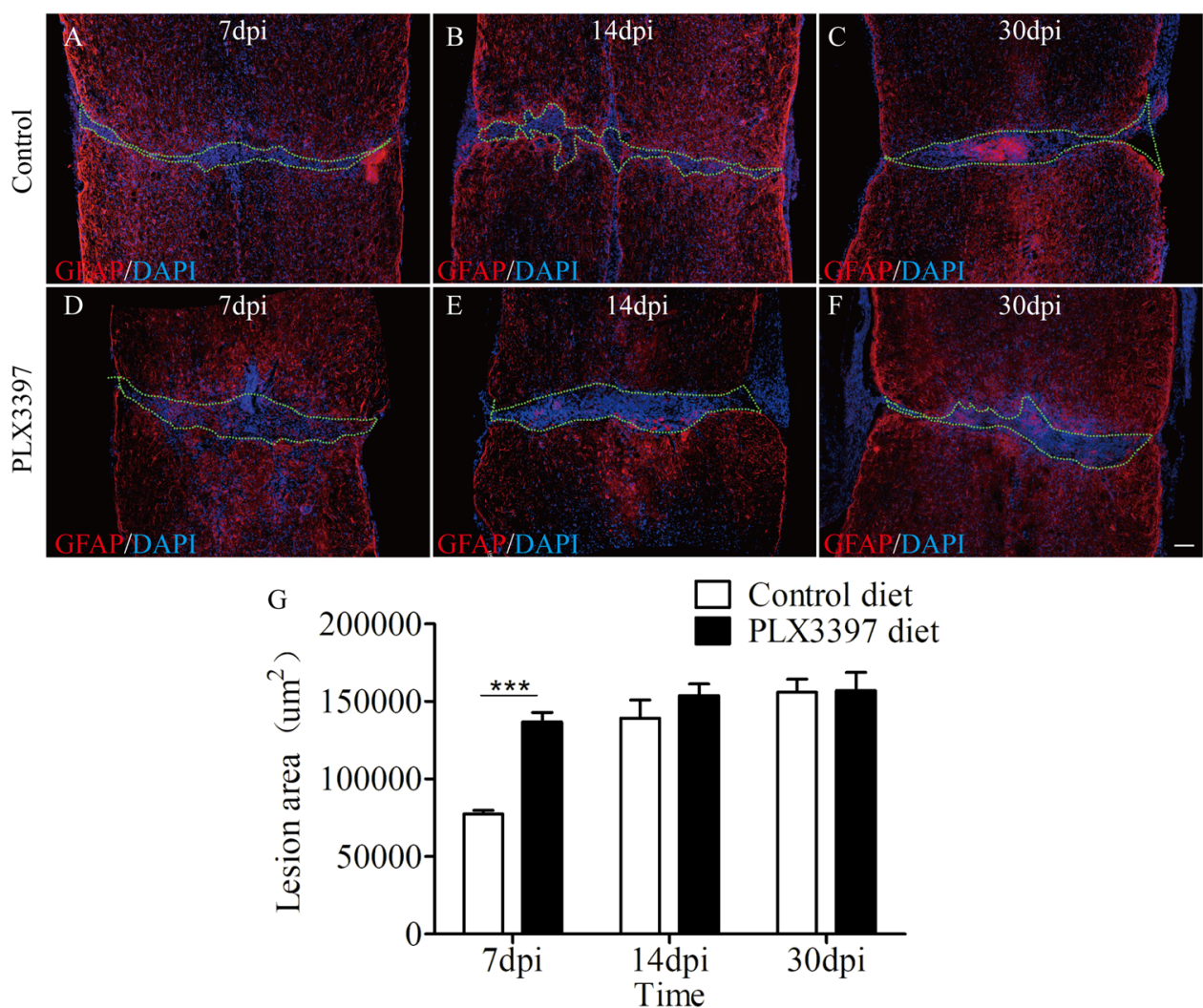

Fig. 6 Delayed repopulation of the spinal cord lesion site by astrocytes upon microglia depletion. a-f Representative photographs of coronal sections of mouse spinal cord taken from mice on either control diet or PLX3397at 7, 14, and $30 \mathrm{dpi}$. The area circled by green dashed line represent lesion area. $\mathbf{g}$ Quantification of the lesion size of the spinal cord from control and PLX3397-treated mice ( $n=4$ per group) as shown in a-f. Data are expressed as mean $\pm S D$. ${ }^{* *} p<0.01$. Scale bars: $\mathbf{a}-\mathbf{f}$, in $\mathbf{f} 100 \mu \mathrm{m}$.

with control diet groups at $30 \mathrm{dpi}$ (Fig. $7 \mathrm{a}-\mathrm{c}$ ). Given microglial depletion delays the repopulation of the lesion site by astrocytes after $\mathrm{SCI}$, and previous study that astrocytes might bridge a lesion site to support axonal regeneration $^{19,21}$, we next analyzed the impact of microglia depletion on axon regeneration in vivo. We performed a full crush SCI at T10 in mice fed with either PLX3397 diet or control diet, injected the axon tracer BDA into the right cortex 6 weeks later, and perfused these mice 8 weeks after SCI. Sagittal sections through the lesion site were stained for BDA to visualize CST axons.
DAPI immunoreactivity was used to visualize cellular nuclei and the lesion. Data revealed that axonal dieback distance in PLX3397-treated mice was significantly increased than that in control treated mice at 8 weeks post SCI (Fig. 7d-f). Altogether, our results indicate that microglia play a neuroprotective role during SCI.

\section{Discussion}

The role of microglia in SCI is still ambiguous because both beneficial and detrimental effects have been proposed. Here, we took advantage of newly developed 

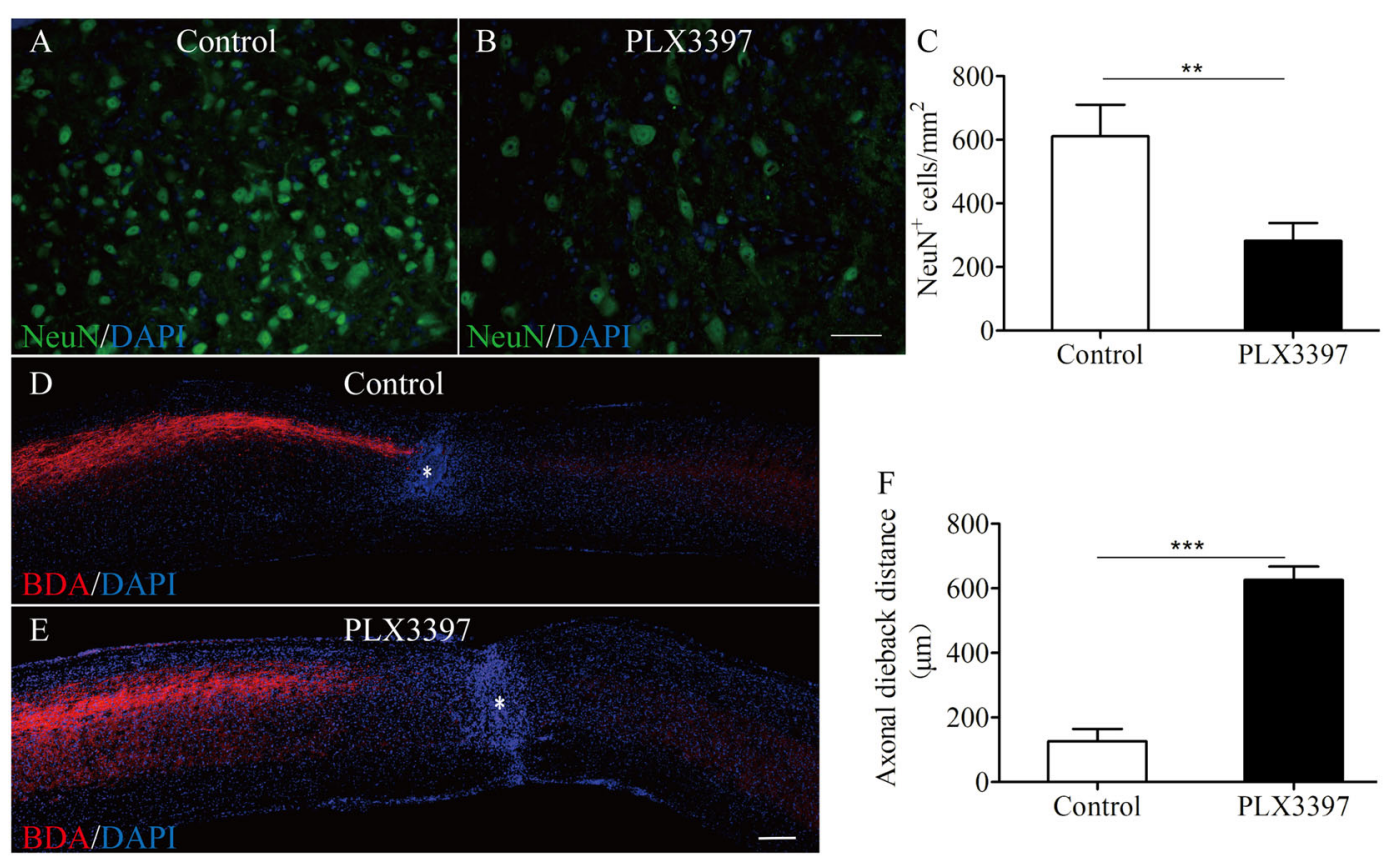

Fig. 7 Microglial depletion reduces neuronal survival and exacerbates axonal dieback. $\mathbf{a}$, $\mathbf{b}$ Representative images taken around the lesion epicenter at $30 \mathrm{dpi}$ immunostained for NeuN (green) and DAPI (blue). c Quantification of the number of $\mathrm{NeuN}^{+}$cells in the spinal cord from control and PLX3397-treated mice ( $n=4$ per group) as shown in $\mathbf{a}$ and $\mathbf{b}$. $\mathbf{d}$, e Representative sagittal sections of the spinal cord from control and PLX3397treated mice at 8 weeks after injury. BDA-labeled CST axons are shown in red. Blue represents DAPI immunostaining. White asterisk represents lesion epicenter. $\mathbf{f}$ Quantification of axonal dieback distance at 8 weeks post SCl as shown in $\mathbf{d}$ and $\mathbf{e}(n=4$ per group). Data are expressed as mean \pm SD. ${ }^{* *} p<0.01,{ }^{* * *} p<0.001$. Scale bars: $\mathbf{a}, \mathbf{b}$, in $\mathbf{b} 50 \mu \mathrm{m} ; \mathbf{d}$, e, in e $200 \mu \mathrm{m}$.

pharmacological depletion strategies that allow us to target microglia specifically to investigate their role in mouse SCI. We demonstrated that PLX3397 efficiently and specifically depleted spinal microglia without any discernable effect on other cell populations in spinal cord. In the context of traumatic SCI, PLX3397 also could efficiently reduce microglial accumulation induced by SCI. Notably, the near-complete microglial elimination by PLX3397 treatment led to the disruption of glial scar formation, which was accompanied by an increased infiltration of blood-derived inflammatory cells into the spinal cord parenchyma. Upon a full crush injury, microglia depletion delayed the repopulation of the spinal lesion site by astrocytes, reduced neuronal survival, exacerbated axonal dieback, and finally impaired locomotor function recovery compared to controls.

Previous studies demonstrated that oral administration of CSF1R inhibitor efficiently resulted in elimination of $\sim 90 \%$ of all microglia brain-wide ${ }^{8,12,22}$. This newly developed depletion strategies were more efficient than a genetic approach, by which diphtheria toxin has to be injected into transgenic mice to induce cell death ${ }^{23}$. Treatment with CSF1R inhibitor also avoided the occurrence of the undesired cytokine storm, a massive increase in cytokine and chemokine production in genetic model ${ }^{24}$. As observed in these studies on brain, we found that continuous treatment with the CSF1R inhibitor PLX3397 also resulted in the depletion of spinal cord microglia (93\%), because almost no Iba1+ cells were detected in spinal cord. Additionally, other cell types in spinal cord, such as neurons, astrocytes, and oligodendrocyte also remained unaffected upon PLX3397 treatment. Notably, treatment times might possibly be reduced from 3 weeks to 1 week, because we found that 1 week of PLX3397 diet was sufficient to achieve near-complete depletion, similar to previous studies in brain or optic nerves ${ }^{5,12}$.

SCI triggers a robust inflammatory response that includes the rapid activation and migration of microglia around the lesion site. The current study consists with this process and Iba1+ cells were also not detectable in spinal cord of injured mice fed with PLX3397 diet. Thus, PLX3397 is similarly effective in the uninjured and injured spinal cord. All these make pharmacological treatments with CSF1R inhibitor become a better method to investigate the specific role of microglia in SCI.

Microglial activation occurs very early after CNS injury. Because of the multiple potentially conflicting capabilities of activated microglia, their overall impact on CNS recovery remains debatable ${ }^{2}$. Traditionally, activated microglia are generally thought to be detrimental to CNS recovery following injury, because the release of proinflammatory cytokines might convey neurotoxicity to 
injured and healthy neurons ${ }^{25,26}$. Upregulation of neurotoxic genes in microglia was detected as early as 1 day post $\mathrm{SCI}^{27}$. Liddelow and his colleagues ${ }^{5}$ showed that activated microglia secrete interleukin (IL)- $1 \alpha$, tumor necrosis factor alpha (TNFo), and C1q, and these cytokines together induce neurotoxic reactive astrocytes, which lose many normal astrocyte functions and induce death of neurons. Accordingly, administration of neutralizing antibodies to IL- $1 \alpha$ or TNF $\alpha$ reduces neuronal cell death and improves functional recovery after $\mathrm{SCI}^{28-30}$. However, the current study standing in contrast to previous discoveries uncovered a rather beneficial impact of activated microglia on SCI because depletion of microglia disrupted glial scar formation, reduced neuronal survival, delayed astrocyte repopulation, exacerbated axonal dieback, and impaired locomotor recovery. Consistent with our results, growing studies reported beneficial effects of microglia in CNS injury ${ }^{8,31-34}$. Especially, one recent study demonstrated that elimination of microglia via administration of the dual CSF1R/c-Kit inhibitor PLX3397 exacerbates postischemic inflammation and brain injury ${ }^{31}$. This again demonstrated that the overall effect of activated microglia in CNS injury is beneficial.

As the most abundant cell type in the spinal cord, astrocytes provide trophic support for neurons, promote synapse formation, maintain homeostasis ${ }^{35,36}$. Astrocytes respond to CNS injury through a process called reactive astrogliosis, and form a glial scar with other cell types, including microglia ${ }^{36}$. Functions of reactive astrocytes have been a matter of some debate, with previous studies showing they are both helpful or harmful to CNS recovery ${ }^{19,36,37}$. Here, we found that microglia regulate the astrocytic response. In the absence of microglia, glial scar formation was perturbed. In line with our results is a previous study, where suppression of microglial proliferation by the cell cycle inhibitor olomoucine attenuated astroglial scar formation after $\mathrm{SCI}^{38}$. This disorganized astroglial scar promotes spread of leukocyte outside of the lesion core, which was accompanied by a reduced neuronal survival, worsened axonal retraction, and attenuated motor recovery. In addition, the repopulation of lesion site by reactive astrocytes was markedly delayed upon microglia depletion. Increased spread of inflammatory cells had also been reported before following selective and conditional ablation of reactive astrocytes after $\mathrm{SCI}^{20}$, as well as in mice with conditional deletion of scar-forming gene from astrocytes ${ }^{39,40}$. Given growing evidence on beneficial roles of reactive astrocytes, we speculate that microglia may provide neuroprotection by modulating astrocyte response after SCI.

However, current data cannot conclude that regulation of the astrocyte response is the only way that contributes to the neuroprotection of microglia after CNS injury. Microglia may play neuroprotection via directly modulating neuronal activity after SCI. As previously mentioned, microglia reduced excitotoxic neuronal injury by regulating intracellular calcium levels ${ }^{8}$, but how microglia could regulate the neuronal activity remains unclear. Jin et al. suggested that conditioned media of microglia could reduce ischemia-induced neural injury, suggesting the possibility that microglia-derived factors may be beneficial for ischemic neurons. Whether this direct interaction between microglia and neurons also exist in context of SCI will certainly need to be investigated in future studies.

In conclusion, our data reveal a neuroprotective role of microglia after SCI. The protection may result from their modulating on the formation of the astroglial scar, and thus sequester blood-derived inflammatory cells in the lesion core so that avoiding inflammation-mediated tissue damage.

\section{Acknowledgements}

This work was supported by National Natural Science Foundation of China (NO. 81520108017, NO. 31872310) and Youth Science Fund Project of the Affiliated Hospital of Qingdao University (NO. 3461).

\section{Author details}

${ }^{1}$ Department of Orthopedics, Chinese PLA General Hospital, Beijing, China. ${ }^{2}$ Department of Orthopaedics, The Affiliated Hospital of Qingdao University, Qingdao, China. ${ }^{3}$ Qingdao Eye Hospital, Shandong Eye Institute, Shandong First Medical University \& Shandong Academy of Medical Sciences, Qingdao, China. ${ }^{4}$ School of Medicine, Nankai University, Tianjin, China

\section{Conflict of interest}

The authors declare that they have no conflict of interest.

\section{Ethics approval and consent to participate}

All animal experiment procedures were conducted with the approval by the Institutional Animal Care and Use Committee of the Chinese PLA General Hospital.

\section{Publisher's note}

Springer Nature remains neutral with regard to jurisdictional claims in published maps and institutional affiliations.

Supplementary Information accompanies this paper at (https://doi.org/ 10.1038/s41419-020-2733-4).

Received: 25 December 2019 Revised: 18 June 2020 Accepted: 22 June 2020

Published online: 13 July 2020

\section{References}

1. Ginhoux, F. et al. Fate mapping analysis reveals that adult microglia derive from primitive macrophages. Science (N. Y., N. Y.) 330, 841-845 (2010).

2. David, S. \& Kroner, A. Repertoire of microglial and macrophage responses after spinal cord injury. Nat. Rev. Neurosci. 12, 388-399 (2011).

3. Nimmerjahn, A., Kirchhoff, F. \& Helmchen, F. Resting microglial cells are highly

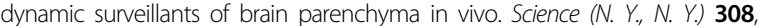
1314-1318 (2005).

4. Davalos, D. et al. ATP mediates rapid microglial response to local brain injury in vivo. Nat. Neurosci. 8, 752-758 (2005). 
5. Liddelow, S. A. et al. Neurotoxic reactive astrocytes are induced by activated microglia. Nature 541, 481-487 (2017).

6. Gerber, Y. N. et al. CSF1R inhibition reduces microglia proliferation, promotes tissue preservation and improves motor recovery after spinal cord injury. Front. Cell. Neurosci. 12, 368 (2018).

7. Tian, D. S. et al. Cell cycle inhibition attenuates microglia induced inflammatory response and alleviates neuronal cell death after spinal cord injury in rats. Brain Res. 1135, 177-185 (2007).

8. Szalay, G. et al. Microglia protect against brain injury and their selective elimination dysregulates neuronal network activity after stroke. Nat. Commun. 7. 11499 (2016)

9. Bellver-Landete, $\mathrm{V}$. et al. Microglia are an essential component of the neuroprotective scar that forms after spinal cord injury. Nat. Commun. 10, 518 (2019).

10. Hilla, A. M., Diekmann, H. \& Fischer, D. Microglia are irrelevant for neuronal degeneration and axon regeneration after acute injury. J. Neurosci.: Off. J. Soc. Neurosci. 37, 6113-6124 (2017).

11. Prinz, M. \& Priller, J. Microglia and brain macrophages in the molecular age: from origin to neuropsychiatric disease. Nat. Rev. Neurosci. 15, 300-312 (2014).

12. Elmore, M. R. et al. Colony-stimulating factor 1 receptor signaling is necessany for microglia viability, unmasking a microglia progenitor cell in the adult brain. Neuron 82, 380-397 (2014)

13. Liu, K. et al. PTEN deletion enhances the regenerative ability of adult corticospinal neurons. Nat. Neurosci. 13, 1075-1081 (2010).

14. Ridnour, L. A. et al. NOS inhibition modulates immune polarization and improves radiation-induced tumor growth delay. Cancer Res. 75, 2788-2799 (2015).

15. Zukor, K. et al. Short hairpin RNA against PTEN enhances regenerative growth of corticospinal tract axons after spinal cord injury. J. Neurosci.: Off. J. Soc Neurosci. 33, 15350-15361 (2013).

16. Basso, D. M. et al. Basso Mouse Scale for locomotion detects differences in recovery after spinal cord injury in five common mouse strains. J. Neurotrauma 23, 635-659 (2006).

17. Neumann, H., Kotter, M. R. \& Franklin, R. J. Debris clearance by microglia: an essential link between degeneration and regeneration. Brain: J. Neurol. 132, 288-295 (2009)

18. Fawcett, J. W. \& Asher, R. A. The glial scar and central nervous system repair. Brain Res. Bull. 49, 377-391 (1999).

19. Anderson, M. A. et al. Astrocyte scar formation aids central nervous system axon regeneration. Nature 532, 195-200 (2016).

20. Faulkner, J. R. et al. Reactive astrocytes protect tissue and preserve function after spinal cord injury. J. Neurosci.: Off. J. Soc. Neurosci. 24, 2143-2155 (2004).

21. Silver, J. \& Miller, J. H. Regeneration beyond the glial scar. Nat. Rev. Neurosci. 5 146-156 (2004)

22. Spangenberg, E. E. et al. Eliminating microglia in Alzheimer's mice prevents neuronal loss without modulating amyloid-beta pathology. Brain: J. Neurol. 139, 1265-1281 (2016).
23. Parkhurst, C. N. et al. Microglia promote learning-dependent synapse formation through brain-derived neurotrophic factor. Cell 155 1596-1609 (2013).

24. Bruttger, J. et al. Genetic cell ablation reveals clusters of local self-renewing microglia in the mammalian central nervous system. Immunity 43, 92-106 (2015).

25. Bosco, A. et al. Reduced retina microglial activation and improved optic nerve integrity with minocycline treatment in the DBA/2J mouse model of glaucoma. Investig. Ophthalmol. Vis. Sci. 49, 1437-1446 (2008).

26. Rice, R. A. et al. Elimination of microglia improves functional outcomes following extensive neuronal loss in the hippocampus. J. Neurosci.: Off. J. Soc. Neurosci. 35, 9977-9989 (2015)

27. Hirbec, E. H., Noristani, H. N. \& Perrin, F. E. Microglia responses in acute and chronic neurological diseases: what microglia-specific transcriptomic studies taught (and did not teach) us. Front. Aging Neurosci. 9, 227 (2017).

28. Nesic, O. et al. IL-1 receptor antagonist prevents apoptosis and caspase-3 activation after spinal cord injury. J. Neurotrauma 18, 947-956 (2001).

29. Ferguson, A. R. et al. Cell death after spinal cord injury is exacerbated by rapid TNF alpha-induced trafficking of GluR2-lacking AMPARs to the plasma membrane. The. J. Neurosci.: Off. J. Soc. Neurosci. 28, 11391-11400 (2008).

30. Genovese, T. et al. TNF-alpha blockage in a mouse model of SCl: evidence for improved outcome. Shock (Augusta, Ga.) 29, 32-41 (2008).

31. Jin, W. N. et al. Depletion of microglia exacerbates postischemic inflammation and brain injury. J. Cereb. Blood Flow. Metab.: Off. J. Int. Soc. Cereb. Blood Flow. Metab. 37, 2224-2236 (2017).

32. Yang, $X$. et al. Depletion of microglia augments the dopaminergic neurotoxicity of MPTP. FASEB J.: Off. Publ. Federation Am. Societies Exp. Biol. 32, 3336-3345 (2018)

33. Lalancette-Hebert, $M$. et al. Selective ablation of proliferating microglial cells exacerbates ischemic injury in the brain. J. Neurosci.: Off. J. Soc. Neurosci. 27 2596-2605 (2007).

34. Lambertsen, K. L. et al. Microglia protect neurons against ischemia by synthesis of tumor necrosis factor. J. Neurosci.: Off. J. Soc. Neurosci. 29, 1319-1330 (2009).

35. Liddelow, S. \& Barres, B. SnapShot: astrocytes in health and disease. Cell 162 1170-1170.e1171 (2015)

36. Sofroniew, M. V. \& Vinters, H. V. Astrocytes: biology and pathology. Acto Neuropathologica 119, 7-35 (2010).

37. Menet, V., Prieto, M., Privat, A. \& Gimenez y Ribotta, M. Axonal plasticity and functional recovery after spinal cord injury in mice deficient in both glial fibrillary acidic protein and vimentin genes. Proc. Natl Acad. Sci. USA 100 8999-9004 (2003).

38. Tian, D. S. et al. Attenuation of astrogliosis by suppressing of microglial proliferation with the cell cycle inhibitor olomoucine in rat spinal cord injury model. Brain Res. 1154, 206-214 (2007).

39. Okada, S. et al. Conditional ablation of Stat3 or Socs3 discloses a dual role for reactive astrocytes after spinal cord injury. Nat. Med. 12, 829-834 (2006).

40. Herrmann, J. E. et al. STAT3 is a critical regulator of astrogliosis and scar formation after spinal cord injury. J. Neurosci.: Off. J. Soc. Neurosci. 28 7231-7243 (2008) 